\title{
Vagal Nerve Schwannoma - A Rare Neoplasm with A Rare Presentation And Newer Surgical Management Technique
}

\author{
Dr. KS Mehta ${ }^{1}$ Dr. Dhruv Gupta ${ }^{2 *}$ Dr. Neeraj Koul ${ }^{3}$ Dr. Deepika Sharma ${ }^{4}$ \\ 1. Professor Department of Surgery ASCOMS and Hospitals Jammu (J\&K) India. \\ 2.Postgraduate Student Department of Surgery ASCOMS and Hospitals Jammu (J\&K) India. \\ *(Corresponding Author) \\ 3.Assistant Professor Department of Surgery ASCOMS and Hospitals Jammu (J\&K) India. \\ 4.Postgraduate Student Department of Surgery ASCOMS and Hospitals Jammu (J\&K) India.
}

\begin{abstract}
Schwannomas are painless, benign, and slow-growing solitary tumors. It involves the cranial nerves such as V, VII, IV, X, XI, and XII or the sympathetic and peripheral nerves and most commonly occur in the neck [1]. These tumors are well encapsulated, and nerve fibers often splay out on the surface but never penetrate the capsule. They have a rare malignant degeneration. Cervicalschwannomas are uncommon, those arising from the cervical Vagal nerve [fig 1,2,3] are extremely rare. We are describing a rare neoplasm cervical vagal schwanoma with a rare presentation of neckmass with chronic cough with newer surgical management technique of nerve preservation which is enucleation and excision.

Key Words: Cervical Vagal Nerve, Schwanoma, Neck Mass,Enucleation, Excision
\end{abstract}

\section{Introduction}

Both benign and malignant tumors may arise from any of the structures contained within the Parapharyngeal space. Of Parapharyngealtumors $70-80 \%$ are benign, and $20-30 \%$ are malignant. Extracranialschwannomas in the head and neck region presenting as parapharyngeal space tumours in absence of neurofibromatosis are rare neoplasms. Schwannoma originating from the cervical vagus nerve is an extremely rare neoplasm [2,3] [fig1,2,3].

Vagal nerve schwannoma usually occurs between the third and fifth decades of life, both sexes being equally affected and it most often presents as a painless, slow-growing, lateral neck mass masses and determination of the nerve origin is not often made until the time of surgery. The treatment of choice is complete surgical excision with preservation of the neural pathway, when ever it is possible, intracapsularenucleation has been introduced for the preservation of the neurological functions.

\section{Case Presentation}

A 25 year old 5 feet 6 inches, $54 \mathrm{~kg}$ unmarried male from bishnahjammu working as dailywager in juice factory reported with a swelling on the right side of neck and dry cough for the past 6 months.He was alright 6 months back when he noticed a swelling on the right side of neck which started suddenly and gradually progressed in size over a period of 6 months to present size of a tennis ball.Patients relatives also noticed a change in character of voice .He also gives history of dry cough for past 6 months which used to occurred through out the day.

On examination $7 \mathrm{~cm}$ by $6 \mathrm{~cm}$ ovoid swelling was found over the right side of the neck, extending vertically from $3 \mathrm{~cm}$ below the mastoid tip to the middle of the posterior border of the sternocliedomastoid muscle and horizontally from $5 \mathrm{~cm}$ behind the angle of the mandible to the midpoint on anterior border of the sternocleidomastoid muscle .It was firm in consistency, non tender, mobile horizontally, margins well defined and over swelling skin normal. Occasionally cough may be produced on palpating the mass . Right carotid artery pulsation was displaced medially and trachea was displaced towards left side. No bruit was noted on auscultation and regional lymph nodes were not enlarged.

Indirect Laryngoscopy-Bilateral vocal cord mobility normal.

Bilateral pyriformfossa clear.

Mild vocal cord congestion.

CECT Neck :-Heterogenous enhancing mass lesion in right retrostyloidparapharyngeal space extending upto carotid bifurcation deep to sternocleidomastoid [fig 4,5A,5B] causing anteromedial displacement of carotid vessels with obscuration of ipsilateral cervical part of internal juglar vein.

Procedure :-Under general anaesthesia,approximately $8 \mathrm{~cm}$ length a cervical incision along the anterior border of the sternocleidomastoid muscle was made and the dissection proceeded beneath the muscle. A yellowish-white, ovoid-shaped mass[fig6,7,8,9] was observed measuring $7 \mathrm{~cm}$ by $6 \mathrm{~cm}$ lying between the carotid artery and the 
internal jugular vein. Mass appeared in continuity with the vagus nerve with no evidence of infiltration. After adequate dissection a plane could be reached,through which it was possible to dissect the splayed nerve trunk off the tumour. The tumour was completely resected using intracapsularenucleation[fig8]after mobilization from the internal jugular vein and carotid artery using microsurgical techniques. The glossopharyngeal, hypoglossal, lingual, accessory nerves were all preserved. Two drain were kept ,one in the cavity and a corrugated drain subcutaneously both of which were removed on $2 \mathrm{nd}$ post operative day and patient dischared on 8th post operative day after stitch removal. Post Operative period[fig 10] was uneventful with no evidence of any cranial nerve paresis ,complaints of cough improved but hoarseness didnt show much improvement post operatively for which patient underwent aggressive voice therapy for 3 months and after which he showed improved speech. The pathological examination confirmed the encapsulated tumour mass a benign schwannoma. Microscopically the neoplasia was composed of spindle cells organized in small fascicles with Antoni-A and Antoni-B cells. Verocay bodies with spindle cells, organized in a palisading fashion all of which was consistent with diagnosis of schwannoma.

\section{Discussion}

Schwannomas are also referred to as neurilemmomas and neuromas. Schwannomas typically present between the forth and sixth decade of life but can occur at any age. Schwanomas are slow growing and rarely cause palsy of the nerve of origin[6]. They are encapsulated and histologically distinct from the nerve itself. Treatment is by enucleation, [fig 6,7,8] and preservation of the nerve of origin if possible.Clinical diagnosis of schwannoma is difficult because many vagal schwannomas do not present with neurological deficits and several differential diagnoses for tumour of the neck may be considered, including paraganglioma,branchial cleft cyst, malignant lymphoma,metastatic cervical lymphadenopathy .

One can assume that tumor arising anterior to styloid process are most likely of salivary gland origin, whereas those of a retrostyloid compartment are vascular or neurogenic.CT scanning can localize the tumour mass in the prestyloid or poststyloidspace[8].

Regardless of the nerve of origin, schwannomas in general are hypodense in relation to muscle tissue on CT without contrast[4]. With contrast, these lesions may show some degree of enhancement [fig5A,5B].Schwannomas of the cervical sympathetic chain were found to displace both the carotid and jugular vessels without separating them[8]. Vagal nerve schwannomas were found to separate the carotid arteries from the internal jugular vein. CT been superseded by MRI [4]with MRI, one can rule out other tumors that can present in asimilar manner and determine the relation of the tumor.Anatomic evaluation by direct vision intraoperatively is an acceptable way but may not be feasible in all cases. Therefore, some diagnoses of the site of origin of schwannoma are definitive other time presumptive. The useful of fine-needle aspiration and cytology is still controversial[2]. Incisional biopsy will obliterate tissue plane thus make removal of tumour mass difficult. In our case patient had undergone prior fine needle aspiration and no conclusive diagnosis could be obtained. Treatment of vagal nerve tumours is a complete surgical excision because they are relatively radioresistant. Intracapsularenucleation has been introduced topreserve the neurological functions[7], which has been classically described as a cautious surgical dissection with extracapsular "peeling". Intracapsularenucleation[fig8] preserves the nerve fibres function more than $30 \%$ when compared to tumour resection with primary anastomosis Post operative Vocal cord palsy has been reported as high as $80 \%$ in the literature[5].Other common complications include pharyngolaryngeal anesthesia, aspiration and cranial nerve IX, X, XII palsies, which maybe transient or permanent.

\section{Conclusion}

Cervical Vagal schwannomas are rare tumours that most often present as asymptomatic unilateral neck masses that progresses very slowly. Definitive preoperative diagnosis may be difficult despite a variety of available imaging techniques. Furthermore, determination of the nerve of origin often remainselusive, even with the combination of CT and MRI the patient's family, should be informedabout the possible post-operative neurological complications.Surgical resection may cause fatal nerve damage unlike other tumors. Therefore, treatmentsassuring the preservation of neurological functions are recommended.

DrDeepika Sharma

\section{Acknowledgments}

Postgraduate Student Surgery ASCOMS

All financial support for the study was done by DrDhruv Gupta and DrDeepika Sharma.

\section{Ethical approval statement}

Written informed consent was obtained from the patient forpublication of this case report and accompanying images. A copy of the written consent is available for review by the Editor-in-Chief of this journal on request. 


\section{References}

[1]. Colreavy MP, Lacy PD, Hughes J, Bouchier-Hayes D, BrennanP, O’Dwyer AJ, et al. Head and Neck schwannomas - a10-year review.JLaryngolOtol 2000;114:119-24.2

[2]. Ford LC, Cruz RM, Rumore GJ, Klein J. Cervical cystic schwannoma of the vagusnerve:Diagnostic and surgicalchallenge. J Otolaryngol 2003;32:61-3. 3

[3]. Green JD, Olsen KD, DeSanto LW, Scheithauer BW (1988)Neoplasms of the vagus nerve. Laryngoscope 98:648-654

[4]. Som P, Sacher M, Stollman A, Biller H, Lawson W (1988)Common tumors of theParapharyngeal space: refined imagingdiagnosis. Radiology 169:81-855.

[5]. Al-Ghamdi S, Black MJ, Lafond G. Extracranial head and neck schwannomas. J Otolaryngol 1992 Jun; 21(3)

[6]. 6.Park CS, Suh KW, Kim CK. Neurilemmomas of the cervical vagus nerve. Head Neck1991;15:439-4111

[7]. St Pierre S, Theriault R, Leclerc JE. Schwannomas of the vagus nerve in the head and neck. J Otolaryngol 1985;14:167-70.

[8]. Furukawa M, Furukawa MK, Katoh K, Tsukuda M. Differentiation between schwannoma ofthevagus nerve and schwannoma of the cervical sympathetic chain by imaging diagnosis.Laryngoscope 1996;106:1548-52

Preoperative Images-

Fig 1

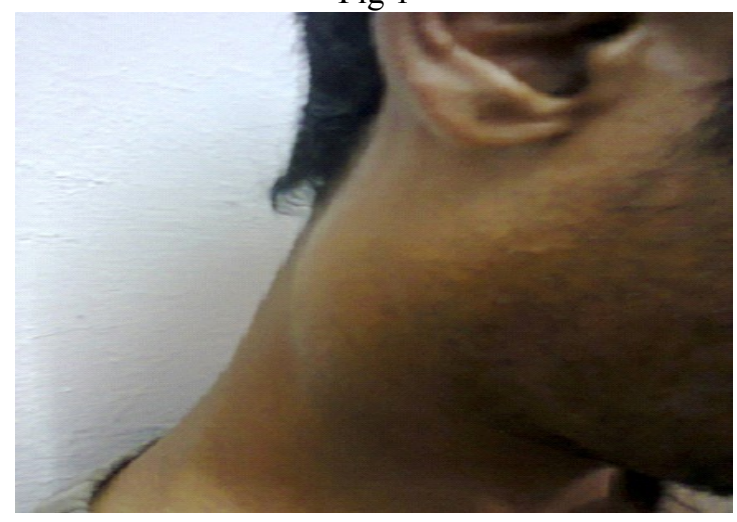

Fig 2

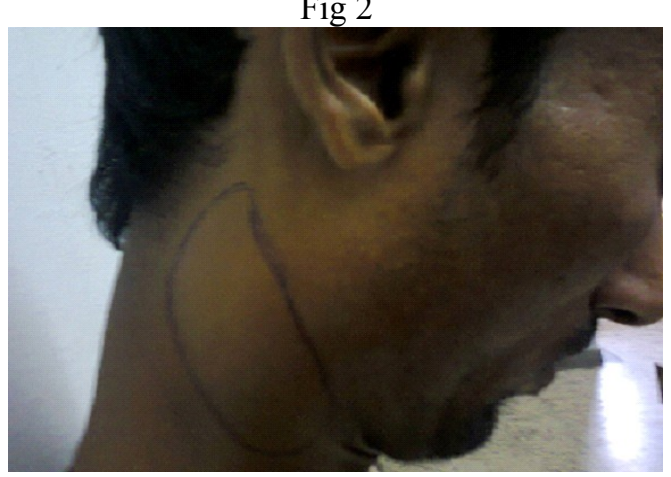

Fig 3

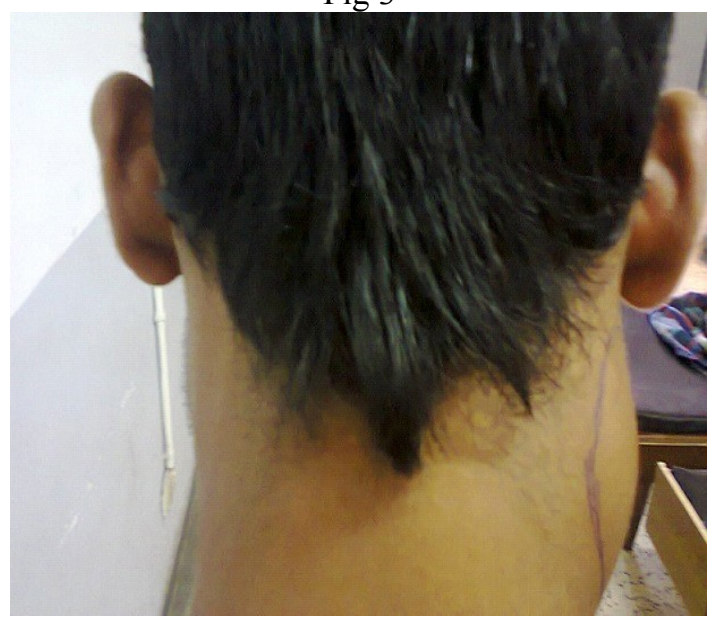


CECT Neck-

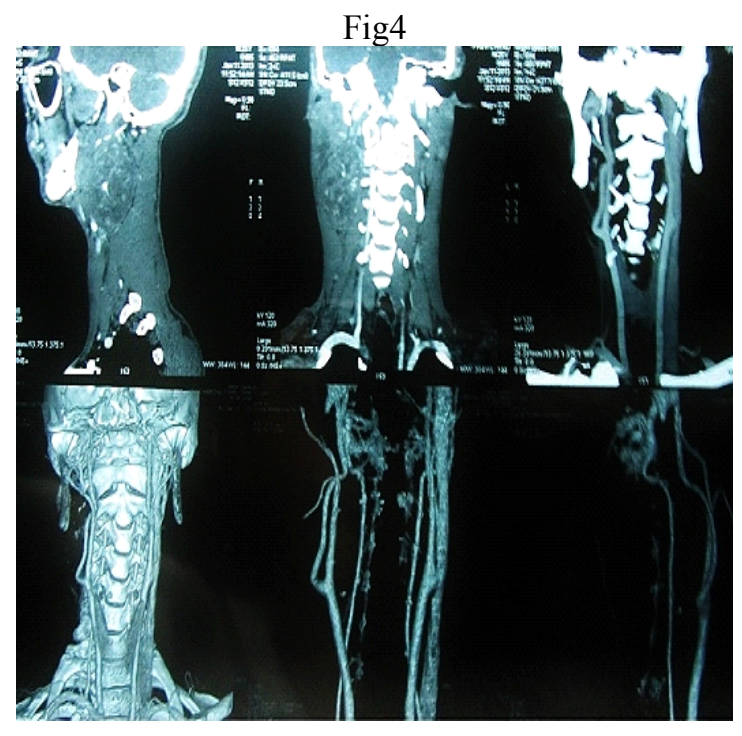

Fig 5A\&5B
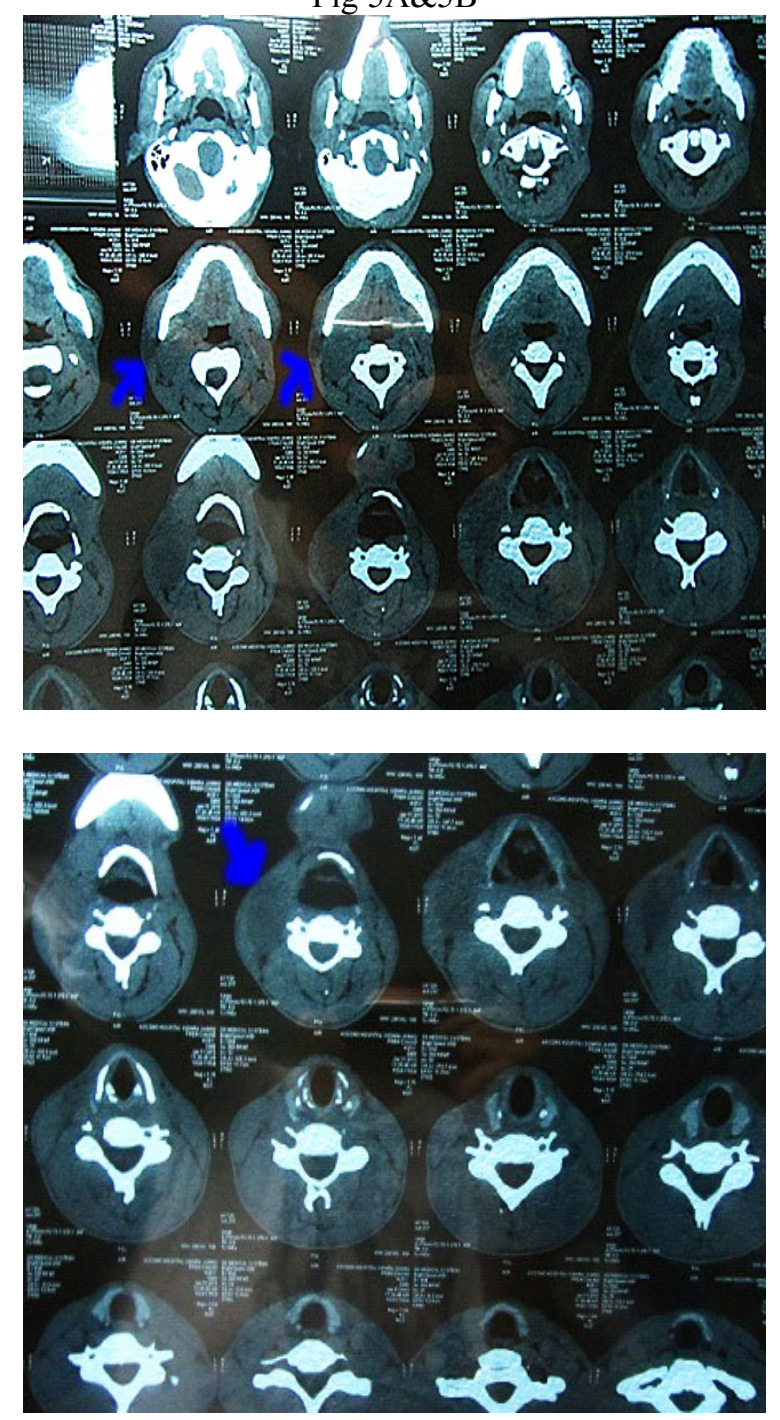
Intraoperative Images

Fig 6

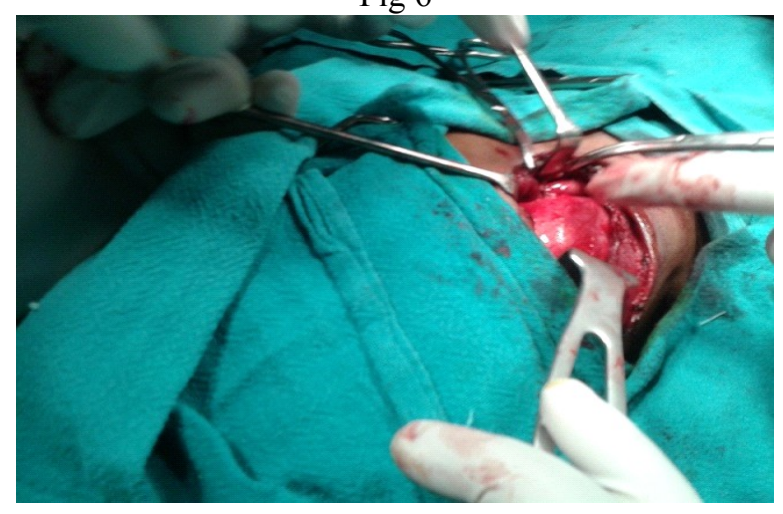

Fig 7

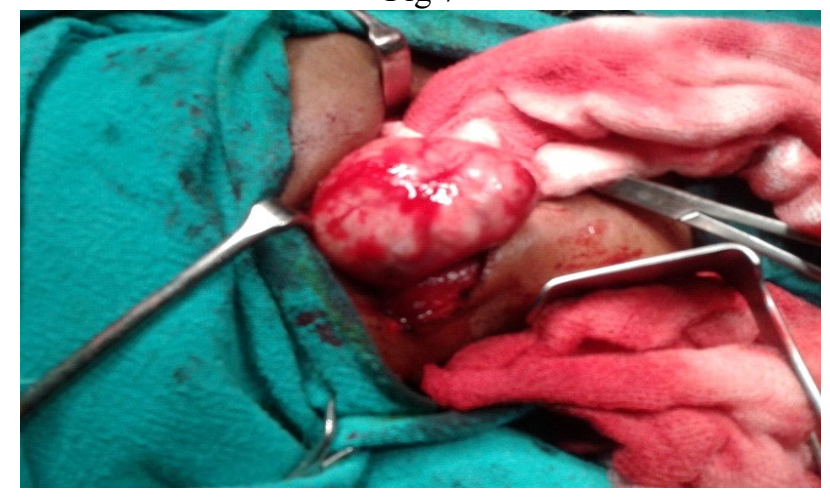

Fig8

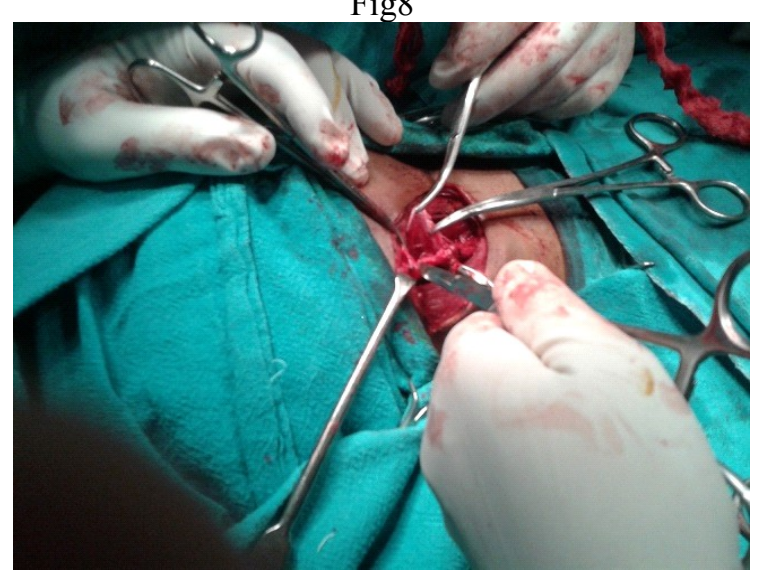

Specimen of tumour mass Fig 9

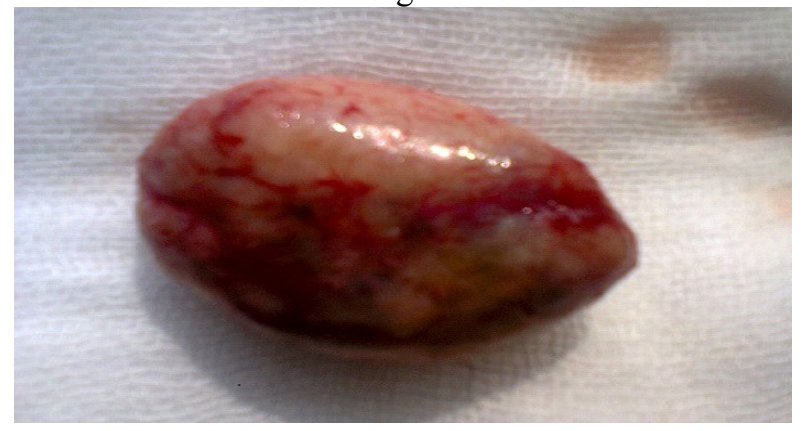


Postoperative (After 2 Weeks)

Fig10

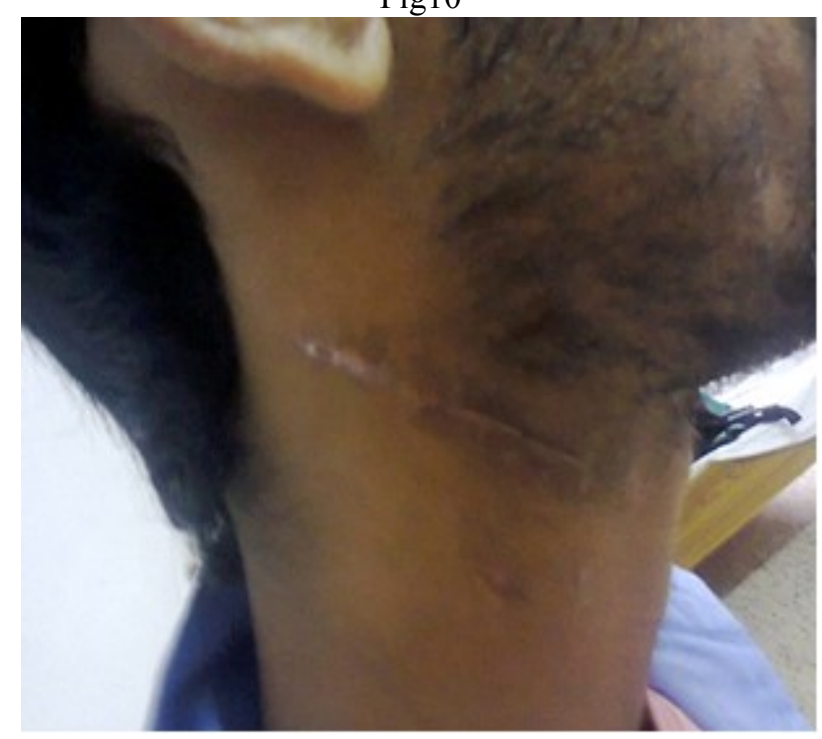

